\title{
Experimental and theoretical analysis of white- light seeded, collinear phase-matching, femtosecond optical parametric amplifiers
}

\author{
Jiun-Cheng Wang \\ Graduate Institute of Electro-Optical Engineering, National Taiwan University, Taipei, Taiwan
}

Juen-Kai Wang

Center for Condensed Matter Sciences, National Taiwan University, Taipei, Taiwan, and Institute of Atomic and Molecular Sciences, Academia Sinica, Taipei, Taiwan

Received April 3, 2003; revised manuscript received September 4, 2003; accepted September 9, 2003

\begin{abstract}
We report a high-power, kilohertz, collinear phase-matching ultrafast optical parametric amplifier (OPA) that is capable of producing $70-\mu \mathrm{J}, \sim 150$-fs infrared laser pulses at wavelengths ranging from 2.9 to $4.0 \mu \mathrm{m}$. The OPA system was seeded with a broadband white-light continuum, which was carefully characterized experimentally. The retrieved electric field of the white-light seed pulse was incorporated in a simulation. The simulated results almost perfectly matched the experimental results of our OPA system. We used the simulation further to investigate the interplay between material dispersion and optical nonlinearity in ultrafast OPA systems and to examine the role of white-light seed pulses in such systems. (C) 2004 Optical Society of America

OCIS codes: $000.4430,190.4970,190.7110$.
\end{abstract}

\section{INTRODUCTION}

A wavelength-tunable mid-IR femtosecond laser source is essential in the investigation of vibrational dynamics in chemical and biological systems and of intrasubband carrier dynamics in semiconductors. In chemical systems the fundamental stretching frequencies for $\mathrm{O}-\mathrm{H}, \mathrm{N}-\mathrm{H}$, $\mathrm{C}-\mathrm{H}$, and $\mathrm{S}-\mathrm{H}$ bonds are of utmost importance and thus require laser pulses that are tunable from 3 to $5 \mu \mathrm{m}$. In particular, sum-frequency generation (SFG) spectroscopy is a unique technique for the investigation of molecular vibrations at surfaces and interfaces. ${ }^{1,2}$ It requires, however, at least $\sim 10-\mu \mathrm{J}$ infrared pulses to produce sufficient vibrational excitation at surfaces and interfaces in timeresolved SFG experiments. The most popular method for generating IR femtosecond light pulses is optical parametric generation. ${ }^{3}$ There are several configurations that can achieve optical parametric generation,, 45 among which optical parametric amplification (OPA) with collinear phase matching possesses the advantage of generating high-power, nearly transform-limited ultrashort laser pulses with an extensive wavelength tuning range. 6,7 Femtosecond pulses at wavelengths longer than $3 \mu \mathrm{m}$ with output pulse energy of $>10 \mu \mathrm{J}$ have been produced by various nonlinear optical crystals. ${ }^{7-12}$ Tunable pulses shorter than $25 \mathrm{fs}$ have been achieved. ${ }^{13}$ The tuning range of collinear phase-matched OPA can be further extended into UV and far IR with the combination of harmonic and difference-frequency mixing. ${ }^{5,8}$ In particular, OPA systems with broadband white-light continua have been successfully demonstrated ${ }^{12,14,15}$ and have been routinely used in various time-resolved spectroscopic experiments. Here we demonstrate that our $1-\mathrm{kHz}$ ultrafast
OPA system is capable of producing $\sim 150$-fs mid-IR laser pulses at wavelengths ranging from 2.9 to $4.0 \mu \mathrm{m}$, with energy as high as $70 \mu \mathrm{J}$.

A theoretical derivation of the nonlinear optical interaction among three electromagnetic waves was given by Armstrong et al. ${ }^{16}$ in 1962 . Because of the complexity of this interaction, simplifications usually were adopted to reveal essential physics. ${ }^{16,17}$ These simplifications, though they are helpful for intuitive understanding, have become inapplicable for contemporary high-power ultrafast OPA systems. Numerical simulations that include material dispersion, field depletion, and other relevant effects have been performed for ultrafast OPA. ${ }^{18-22}$ In particular, Bakker et al. ${ }^{18}$ presented a modified Runge-Kutta method and quantitatively studied the effects of group-velocity mismatch (GVM), phase mismatch, and field depletion on laser pulses in second-order optical processes. ${ }^{18}$ Gale et al. further incorporated groupvelocity dispersion (GVD) and third-order nonlinearities to compare their simulation results with experimental measurements. ${ }^{19,20}$ They successfully incorporated cascaded second-order optical nonlinearity in simulations to explain pulse self-compression behavior.

Though there have been many theoretical studies of the subject, our understanding of this ultrafast optical technology is still incomplete. For example, how do material dispersion and second-order optical nonlinearity in the OPA process interplay to give the final pulse shape and energy? What is the role of the seed pulse? Here we report a numerical simulation of collinear phase-matching femtosecond OPA seeded by a white-light continuum. As in previous theoretical efforts, field depletion, GVM, GVD, 
nonlinear refraction, and linear absorption are properly incorporated. In our study, an actual white-light seed pulse and finite phase-matching bandwidth were taken into account. The white-light seed pulse was characterized experimentally, and its electric field was retrieved by a phase-retrieval algorithm. The validity of our simulation was verified by our actual OPA system. We demonstrated the versatility of our simulation as a useful tool in designing and optimizing OPA systems. ${ }^{23}$ In this research the simulation was used as an analytical tool with which to understand the interaction between material dispersion and nonlinear polarization, which cannot be revealed by experimental diagnostic techniques.

In Section 2 we describe the experimental setup of the OPA system and the apparatus used to characterize its output. An experimental characterization of the whitelight continuum is also presented. A mathematical model of three-wave interaction, third-order nonlinear optical interaction, and other effects in simulating the OPA system is described in Section 3. In Section 4 we present both experimental and simulation results. First we show the results of our efforts to characterize the white-light seed pulse. The simulation results are compared with the experimental measurements of the OPA system. Analysis of the pulse evolution behavior during OPA is emphasized. Furthermore, the influence of fluctuations in the pump intensity and the use of an unrealistic whitelight seed beam are analyzed in detail. Finally, Section 5 concludes this paper.

\section{EXPERIMENTS}

The pump source used in our OPA system consists of a mode-locked Ti:sapphire laser pumped by a cw diodepumped Nd: $\mathrm{YVO}_{4}$ laser and of a Ti:sapphire regenerative amplifier system pumped by the second harmonics of two cw $Q$-switched Nd:YLF lasers. The resultant output consists of $1-\mathrm{kHz}, 3-\mathrm{mJ}$, and $\sim 100-\mathrm{fs}$ pulses with wavelengths centered at $800 \mathrm{~nm}$. A single-shot autocorrelator is used to monitor the pulse duration and the stability of the regenerative amplifier output pulses $(<1.5 \%$, pulse to pulse). The whole laser system can maintain its stability within $3 \%$ over more than $10 \mathrm{~h}$, which greatly facilitates our characterization of the OPA system described below. Ninety percent of the output energy is used to pump the OPA system. The remainder is used to characterize the pulse duration of the idler wave in a cross-correlation experiment. The pump beam is further split with a 50/50 beam splitter to give $\sim 1.3-\mathrm{mJ}$ of energy for each pulse. Figure 1 depicts the home-built OPA system. It consists of three amplification stages and two $\mathrm{KTiOAsO}_{4}$ (KTA) nonlinear optical crystals. A small portion of the first pump beam (95/5 beam splitter, BS1) is focused onto a 2-mm sapphire plate to generate a white-light seed beam (white-light generator), which is then directed via delay line D1 onto the first KTA crystal (KTA1). The combination of a zero-order half-wave plate and a thin-film polarizer is used to fine tune the input energy in the whitelight generator. An iris diaphragm is used to adjust the self-focusing condition in the sapphire plate. A major portion of the first pump beam is split into two beams by a 15/85 beam splitter (BS2). The smaller reflected beam

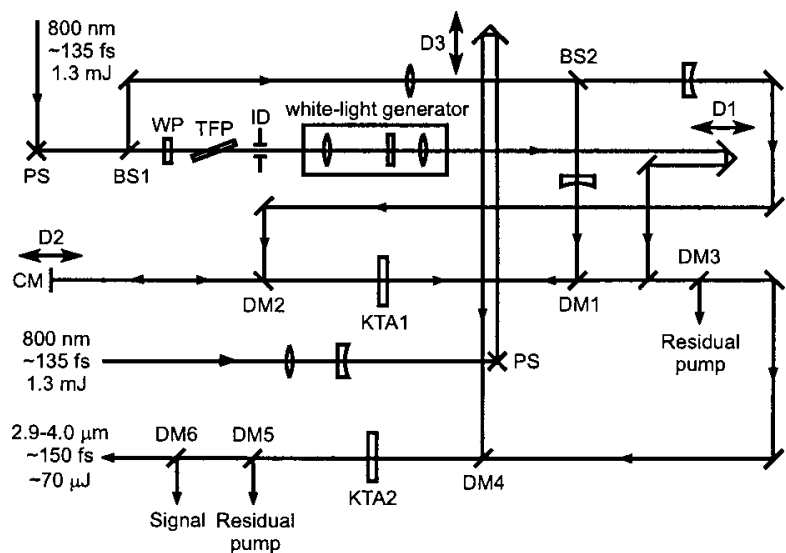

Fig. 1. Schematic of the OPA system: PS's, periscopes; BS1, BS2, beam splitters $(R / T=95 / 5,15 / 85$, respectively); WP, a zero-order half-wave plate; TFP, thin-film polarizer; ID, iris diaphragm; CM, concave Ag mirror. The white-light generator consists of two convex lenses and a 2-mm sapphire plate. KTA1, the KTA nonlinear optical crystal for the first and second amplification stages; KTA2, that for the third amplification stage. Other abbreviations defined in text.

is made collinear with the white-light seed beam and directed onto KTA1 by dichroic mirror DM1. This is the first amplification stage. The residual pump beam is then removed by dichroic mirror DM2. The generated IR beams (signal and idler) are reflected off a concave Ag mirror to become collimated and are then directed onto KTA1 but slightly vertically displaced. The larger portion $(85 \%)$ of the first pump beam is combined with the reflected IR beams by dichroic mirror DM2. The temporal overlap was controlled by another delay line (D2). This is the second amplification stage. The residual pump beam is removed by dichroic mirror DM3 similarly, and the IR outputs of the second amplification stage are directed onto the second KTA crystal (KTA2). The second pump beam is combined with the IR output of the second amplification stage by dichroic mirror DM4. The resultant beam is subsequently sent to the third amplification stage. Similarly, temporal overlap is controlled by a third delay line (D3). After that, the residual pump beam and the generated signal beam are removed by dichroic mirrors DM5 and DM6, respectively. The two KTA crystals are cut for type II phase matching at $\theta=43^{\circ}$ and $\phi=0^{\circ}$. The light waves propagate in the $x z$ plane. The signal wave (extraordinary) is polarized in the $x z$ plane. Both the pump and the idler waves (ordinary) are polarized along the $y$ axis. The crystal thickness is 3 $\mathrm{mm}$, and the crystal surfaces are antireflection coated at $800 \mathrm{~nm}$ to minimize the reflection loss of the pump beam.

The average power of the IR output was measured by a thermopile detector. The spectral characterization was performed with a $30-\mathrm{cm}$ monochrometer in combination with an InSb detector cooled by liquid $\mathrm{N}_{2}$. A Ge longpass filter in front of the entrance slit of the monochrometer removed all the remaining signal and visible light that accompanied the idler beam. A small portion of the regenerative amplifier output was used to characterize the pulse duration of the idler wave. We performed cross-correlation measurements by producing a reflection SFG signal from a GaAs wafer with the 800-nm regenera- 
tive amplifier output and the idler beam. Because there were no phase-matching constraints, surface reflection SFG was demonstrated to be a useful tool for determining the pulse widths of ultrashort UV and IR laser pulses. ${ }^{24,25}$ In our case, special care was taken to ensure minimum excitation of the GaAs surface during the measurements. Autocorrelation measurement of the $800-\mathrm{nm}$ pulse was performed with a 1-mm, type I phase-matched KDP nonlinear optical crystal. Assuming a Gaussian pulse shape, we extracted the pulse duration of the idler wave from the cross-correlation and autocorrelation traces.

To incorporate the white-light seed pulse into the simulation required full characterization of its electric field (amplitude and phase). Because the idler wave is tunable from 2.9 to $4.0 \mu \mathrm{m}$, corresponding to a seed wavelength range of $1-1.1 \mu \mathrm{m}$, experimental characterization of the white-light continuum was focused on the IR region of the continuum spectrum. The characterization procedure and setup are described below. The white-light continuum was first dispersed by a $0.25-\mathrm{m}$ monochrometer and detected by a calibrated thermoelectrically cooled InGaAs photodiode. The InGaAs photodiode was calibrated by a series of cross-calibration measurements. First, a silicon photodiode was calibrated by a predetermined energy measurement at $800 \mathrm{~nm}$. Second, we achieved the response calibration of the InGaAs photodiode by matching the measured signals of the white-light continuum at $980 \mathrm{~nm}$ by the two photodiodes. The spectral energy density of the white-light continuum was then measured. For the temporal information, the white-light continuum and a small portion of the amplifier output were focused noncollinearly onto a $1-\mathrm{mm} \beta-\mathrm{BaB}_{2} \mathrm{O}_{4}$ crystal (type I phase matching) to produce a SFG generation signal. The cross-correlation traces were then recorded. Although the spectrum of the white light spread for several hundreds of nanometers, only a small portion of the whole spectrum was used as the seed in these ultrafast OPA systems. Here, the relevant wavelength range of the measured energy density spectrum was selected by an artificial bandpass filter, which was a spectral boxcar centered at $\sim 1050 \mathrm{~nm}$ with an adjustable spectral bandwidth. Its short- and long-wavelength cutoff edges were adjustable Gaussian-shaped tails. Based on the modified energy density spectrum and the cross-correlation traces, we constructed a cross-correlation frequency-resolved optical gating (XFROG) trace. With the pulse shape of the $800-\mathrm{nm}$ pulse determined by the autocorrelation measurement, the electric field of the white-light seed pulse was retrieved with a phase-retrieval algorithm. The bandwidth of the artificial filter was adjusted iteratively until it covered the whole relevant signal wavelength range, and a wider bandwidth produced only a negligible variation in the retrieved results.

\section{NUMERICAL SIMULATION}

In deriving the nonlinear coupling equations for optical parametric generation, we made two assumptions. First, the electromagnetic fields were assumed to be quasimonochromatic, so the slowly varying envelope approximation could be applied. Second, the electromagnetic fields were assumed to be plane waves, and accordingly the transverse variation of the fields was ignored. These two conditions were satisfied primarily because the pulse duration of the pump laser was $\sim 135$ fs and only a moderate focusing setup was used, such that the depth of focus was longer than the crystal thickness. With these assumptions, the complex electric field can be written as follows:

$$
E_{j}(z, t)=A_{j}(z, t) \exp \left[i\left(k_{j} z-\omega_{j} t\right)\right]+\text { c.c. }
$$

where $j=P, S, I$ stand for pump, signal, and idler waves, respectively, $A_{j}(z, t)$ is the field envelope, $k_{j}$ is the wave vector, and $\omega_{j}$ is the carrier frequency of wave $j$. In the collinear phase-matching configuration the nonlinear coupling equations can be derived as ${ }^{3,19}$

$$
\begin{aligned}
\frac{\partial B_{P}(z, \omega)}{\partial z}= & \frac{i}{2} \omega^{2} \frac{\partial^{2} k_{P}}{\partial \omega^{2}} B_{P}(z, \omega)-\frac{\alpha_{P}}{2} B_{P}(z, \omega) \\
& +\frac{i \omega_{P}}{n_{P} c} P_{P} \mathrm{NL}_{(}(z, \omega), \\
\frac{\partial B_{S}(z, \omega)}{\partial z}=i \omega\left(\frac{1}{v_{S}}-\frac{1}{v_{P}}\right) B_{S}(z, \omega) & +\frac{i}{2} \omega^{2} \frac{\partial^{2} k_{S}}{\partial \omega^{2}} B_{S}(z, \omega)-\frac{\alpha_{S}}{2} B_{S}(z, \omega) \\
& +\frac{i \omega_{S}}{n_{S} c} P_{S} \mathrm{NL}_{(}(z, \omega), \\
\frac{\partial B_{I}(z, \omega)}{\partial z}=i \omega\left(\frac{1}{v_{I}}-\frac{1}{v_{P}}\right) B_{I}(z, \omega) & \\
+ & \frac{i}{2} \omega^{2} \frac{\partial^{2} k_{I}}{\partial \omega^{2}} B_{I}(z, \omega)-\frac{\alpha_{I}}{2} B_{I}(z, \omega) \\
+ & \frac{i \omega_{I}}{n_{I} c} P_{I}{ }^{\mathrm{NL}}(z, \omega) .
\end{aligned}
$$

Here we used retarded time coordinates for the pump wave. $B_{j}(z, \omega)$ is the Fourier transform of field amplitude $A_{j}(z, t) . \quad n_{j}$ is the refractive index, $\alpha_{j}$ is the linear absorption coefficient, $v_{j}$ is the group velocity, and $\partial^{2} k / \partial \omega^{2}$ is the group-velocity dispersion of wave $j$ at $\omega_{j}$. $c$ is the speed of light in vacuum.

Interactions among the three waves are described by the nonlinear polarization $\left\{P_{j}^{\mathrm{NL}}\right\}$. In the simulation we consider second- and third-order nonlinear polarization and, therefore, $P_{j}{ }^{\mathrm{NL}}=P_{j}{ }^{(2)}+P_{j}{ }^{(3)}$. The second-order nonlinear polarizations are expressed as follows:

$$
\begin{aligned}
P_{P}^{(2)}(z, \omega)= & \epsilon_{0} \chi_{\mathrm{eff}}^{(2)}\left[\int \mathrm{d} \omega^{\prime} F_{S}\left(\omega^{\prime}\right) F_{I}\left(\omega-\omega^{\prime}\right)\right] \\
& \times \exp \left[-i k_{P}(\omega) \Delta z\right], \\
P_{S}^{(2)}(z, \omega)= & \epsilon_{0} \chi_{\mathrm{eff}}^{(2)}\left[\int \mathrm{d} \omega^{\prime} F_{P}\left(\omega^{\prime}\right) F_{I}\left(\omega-\omega^{\prime}\right)\right] \\
& \times \exp \left[-i k_{S}(\omega) \Delta z\right],
\end{aligned}
$$




$$
\begin{aligned}
P_{I}^{(2)}(z, \omega)= & \epsilon_{0} \chi_{\mathrm{eff}}^{(2)}\left[\int \mathrm{d} \omega^{\prime} F_{P}\left(\omega^{\prime}\right) F_{S}\left(\omega-\omega^{\prime}\right)\right] \\
& \times \exp \left[-i k_{I}(\omega) \Delta z\right],
\end{aligned}
$$

where $F_{j}(z, \omega)=B_{j}(z, \omega) \exp \left[i k_{j}(\omega) \Delta z\right] . \quad \epsilon_{0}$ is the electric permittivity of vacuum, $\chi_{\text {eff }}^{(2)}$ is the effective secondorder nonlinear susceptibility, and $\Delta z$ is the increment of propagation distance. The derivation is based on the fact that the finite spectral widths of ultrafast laser pulses need to be considered in calculations of nonlinear polarization. The output field is the sum of all the generated frequency components that satisfy the energy conservation relation $\omega_{P}=\omega_{S}+\omega_{I}$. Accordingly, many such frequency combinations can generate a specific frequency, and they should have different gains. The expressions in Eqs. (5)-(7) therefore take the finite spectral widths of ultrashort laser pulses into proper account. More importantly, they endow the nonlinear optical crystal at a specific orientation with the ability to amplify the corresponding wavelength in the white-light spectrum, providing wavelength tunability in the OPA process. Notice that Eqs. (5)-(7) can be simplified to those used by other groups of researchers when the phase mismatch is assumed to be zero. ${ }^{19,20}$

The third-order nonlinear optical processes in Eqs. (2)(4) are self-phase and cross-phase modulation. Similarly to that of second-order nonlinear polarization, the tensor nature of the third-order nonlinear susceptibility needs to be considered. Because of the lack of experimental values of the $\chi^{(3)}$ tensor elements, Gale et al. obtained approximate third-order nonlinear polarization by assuming an isotropic nonlinear optical crystal. ${ }^{19}$ Here we follow such an approach and express the three third-order nonlinear polarizations that are responsible for self-phase and cross-phase modulation in Eqs. (2)-(4) as follows:

$$
\begin{aligned}
P_{P}{ }^{(3)}(z, \omega)= & \mathrm{FT}\left\{\frac { 3 } { 2 } \chi _ { \mathrm { eff } } ^ { ( 3 ) } A _ { P } \left(\gamma_{P P}\left|A_{P}\right|^{2}+2 \gamma_{P S}\left|A_{S}\right|^{2}\right.\right. \\
& \left.\left.+2 \gamma_{P I}\left|A_{I}\right|^{2}\right)\right\}, \\
P_{S}^{(3)}(z, \omega)= & \mathrm{FT}\left\{\frac { 3 } { 2 } \chi _ { \mathrm { eff } } ^ { ( 3 ) } A _ { S } \left(2 \gamma_{S P}\left|A_{P}\right|^{2}+\gamma_{S S}\left|A_{S}\right|^{2}\right.\right. \\
& \left.\left.+2 \gamma_{S I}\left|A_{I}\right|^{2}\right)\right\}, \\
P_{I}^{(3)}(z, \omega)= & \mathrm{FT}\left\{\frac { 3 } { 2 } \chi _ { \mathrm { eff } } ^ { ( 3 ) } A _ { I } \left(2 \gamma_{I P}\left|A_{P}\right|^{2}+2 \gamma_{I S}\left|A_{S}\right|^{2}\right.\right. \\
& \left.\left.+\gamma_{I I}\left|A_{I}\right|^{2}\right)\right\},
\end{aligned}
$$

where FT stands for a Fourier transform, $\chi_{\text {eff }}^{(3)}$ is the effective third-order nonlinear susceptibility, and $\gamma_{i j}$ is a correction factor that equals 1 when $i=j$ and equals $1 / 3$ when $i \neq j$. Notice that phase mismatching is neglected in Eqs. (8)-(10) because no wavelength conversion is involved in either self-phase or cross-phase modulation and therefore the resultant phase mismatch is much smaller than that in OPA. To determine $\chi_{\text {eff }}^{(3)}$, one has to consider the possibility of cascaded second-order optical nonlinearity. It has been shown that cascade effects are quite influential on output characteristics. ${ }^{19}$ The effective thirdorder nonlinear susceptibility can be an order of magnitude larger than the intrinsic third-order susceptibility. Through careful calculations we determined that no cascade effect occurs over the wavelength tuning range in our OPA system; therefore, with the assumption of an isotropic medium, $\chi_{\mathrm{eff}}^{(3)}$ was taken to be $\chi_{x x x x}^{(3)} \sim 1.8$ $\times 10^{-21} \mathrm{~m}^{2} / \mathrm{V}^{2}$ for KTA crystal. ${ }^{26}$

In the remainder of this section, we illustrate all the simulation conditions that are needed to mimic the operational condition in our OPA system. First the angle of the nonlinear optical crystal is specified. The corresponding center wavelengths of the signal and idler waves are then determined based on the phase-matching requirement. With all the coefficients calculated according to the dispersion relation ( $n$ and $k$ ) of the KTA crystal, ${ }^{27}$ Eqs. (2)-(4) are solved numerically by the fourth-order Runge-Kutta method. ${ }^{28}$ As the phasematching condition is appropriately incorporated into Eqs. (5)-(7), the simulation automatically picks up the desired signal wavelength from the broad white-light continuum spectrum. The output center wavelengths of the signal and idler pulses are therefore a natural outcome of the simulation without any initial assignment of desired output wavelengths. In other theoretical works, perfect phase matching was assumed and a special initial pulse shape was adopted. ${ }^{20-22,29}$ The dichroic mirrors in the OPA system were also carefully considered in the calculation. It should be emphasized that optical properties of the optical components between amplification stages (DM2-DM4) strongly influence the output characteristics of the OPA system. This is so because the pulse duration of the signal and the idler waves is broadened and the temporal delay between them is varied because of the GVD and the GVM of these optical components. Moreover, linear loss of these optical components reduces the energy of the signal and the idler waves before the next amplification stage. The temporal positions of the pump pulse in the first and second amplification stages were adjusted to maximize the summation of the signal and the idler output energies because the total output energy was measured during the alignment. The temporal position of the pump pulse in the third amplification stage was adjusted to maximize only the idler output energy because it was measured in the actual system.

The initial parameters in our simulation were the average energy density of the pump beam at each amplification stage and the white-light seed beam. These values were estimated based on measurements of our OPA system. The energy of the first pump beam (the total amount for the white-light generator and the first and the second amplification stages) and the energy of the second pump beam (for the third amplification stage) were measured to be $\sim 1.3 \mathrm{~mJ}$ before being sent into the OPA system (Fig. 1). We calculated the total energy transmitted to the KTA crystal in each amplification stage by considering the losses induced by the optical components used in our OPA system. The energy of the white-light seed pulse was determined from measurement of the spectral energy density described above. In our simulation the 
diameters of the signal and the idler beams were assumed to be identical and were smaller than that of the pump beam. The beam diameter of the seed beam and the energy density of the pump beam were then adjusted slightly about their experimentally determined values to match the simulated output energy of the idler wave at $\lambda_{\text {idler }}=3.0 \mu \mathrm{m}$ with the experimental value. The pulse shape of the idler wave was monitored simultaneously to ensure its good correspondence with the cross-correlation measurement at $3 \mu \mathrm{m}$. This procedure was carried out at each amplification stage. The final energy densities of the pump beam adopted at the first, second, and third amplification stages were $7.1,1.9$, and $1.3 \mathrm{~mJ} / \mathrm{cm}^{2}$, respectively. The energy density of the white-light seed beam was $1.6 \mu \mathrm{J} / \mathrm{cm}^{2}$. These numbers are very close (within $8 \%$ ) to those estimated from our experimental setup. After the simulation parameters were determined, the simulation was performed over the whole wavelength tuning range of the OPA system without any further adjustment of these parameters. In contrast, large scaling adjustment was needed in previous works. ${ }^{19,29}$

\section{RESULTS AND DISCUSSION}

\section{A. Characterization of the White-Light Seed Pulse}

Although using a white-light continuum to seed the signal wave is popular for many OPA systems, the actual white-light electric field has, to our knowledge, never been incorporated into simulations. The reasons may be that many complicated nonlinear optical processes are involved in supercontinuum generation such that it cannot be derived theoretically, and, more importantly, that no direct experimental characterization has to our knowledge ever been performed. Instead of speculating about the white-light continuum based on existing theories, we made a great effort to retrieve its intensity and phase. The relative group delay and the intensity spectrum of the white-light seed pulse are shown in Fig. 2. Experimental data for the intensity spectrum have been scaled to facilitate a comparison with the retrieved results. Notice that the intensity spectrum decreased almost exponentially with the output wavelength, consistent with other experimental results. ${ }^{7,30}$ Figure 3 compares the

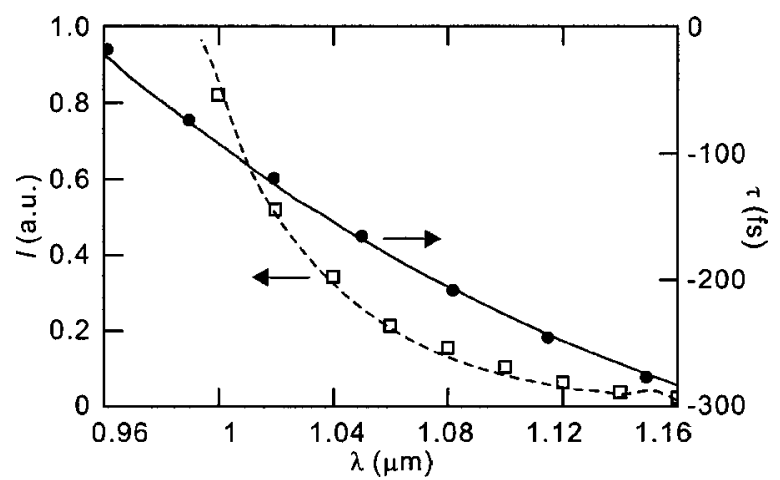

Fig. 2. Relative group delay and intensity spectrum of the white-light seed pulse. Experimental data are represented by symbols; curves are retrieved results. Open squares and the dashed curve, the intensity spectrum; filled circles and the solid curve, the relative group delay.

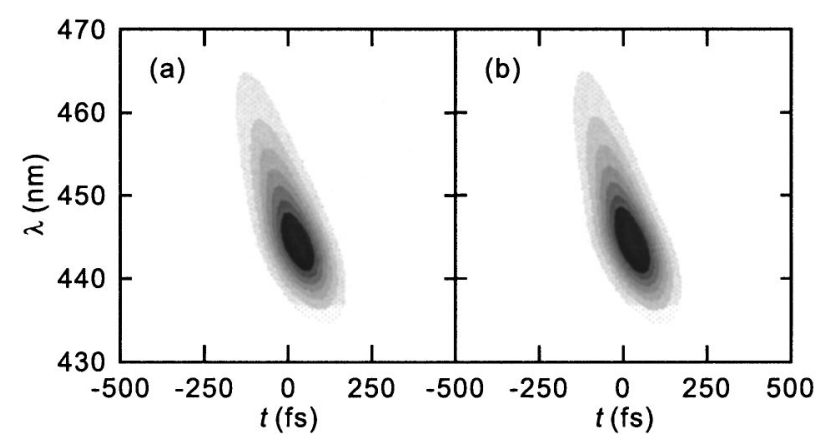

Fig. 3. (a) Constructed and (b) retrieved XFROG traces of the white-light seed pulse.
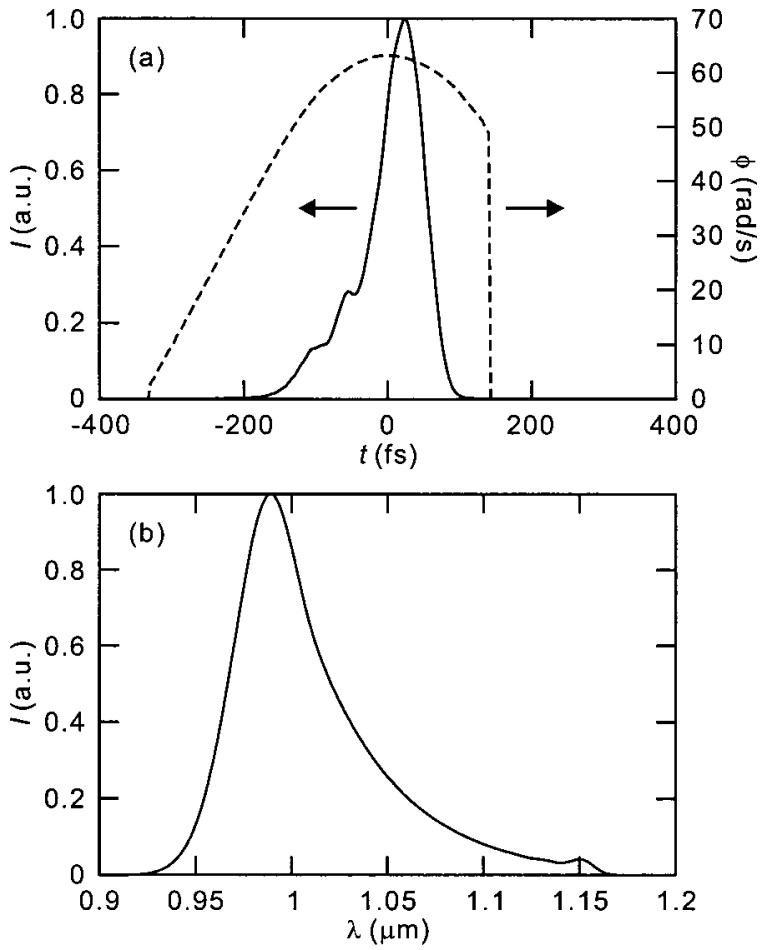

Fig. 4. Retrieved electric field of the white-light seed pulse: (a) retrieved intensity ( $I$, solid curve) and phase ( $\phi$, dashed curve). (b) Intensity spectrum.

constructed and the retrieved XFROG traces of the whitelight seed pulse. Figure 4(a) shows the retrieved intensity and phase in the time domain. Self-steepening and pulse shortening appear in the pulse shape, indicating the occurrence of self-focusing and other higher-order nonlinear optical effects during the generation of a supercontinuum. ${ }^{30}$ The phenomenon of self-steepening was also observed by Fork et al. ${ }^{31}$ and shown in a theoretical calculation by Yang and Shen. ${ }^{32}$ The positive chirp, which can also be recognized from the XFROG traces, was observed experimentally by Diddams et $a l .{ }^{33}$ The linear chirp was determined to be $\sim 7.21 \times 10^{-4} \mathrm{fs}^{-2}$ and is higher than that estimated from the 2-mm sapphire window $\left(\sim 1.7 \times 10^{-4} \mathrm{fs}^{-2}\right)$. Figure 4 (b) shows the retrieved intensity spectrum of the white-light seed pulse. Based on this retrieved electrical field, the energy spectral density and the group delay were calculated as a function of wavelength. The comparison shows an almost perfect 
match (Fig. 2). The cutoffs at both 0.98 and $1.15 \mu \mathrm{m}$ are a consequence of the artificial filter mentioned above. No significant difference in the retrieved results was discovered when the bandwidth of this truncated filter was enlarged. We note that several similar efforts were made recently to retrieve the electric fields of white-light continua generated in photonic crystal optical fibers. ${ }^{34-36}$ The retrieved intensity and phase of the white-light continuum were significantly different from the artificially created ones used in previous simulation efforts. ${ }^{20-22,29}$ This result therefore justifies our effort to retrieve the actual white-light seeded signal pulse and using it in the simulation.

\section{B. Comparison of the Experimental and the Simulation Results}

Figure 5 shows a comparison of the experimental and the simulation results of the output idler pulses. Simulation results with (solid curves) and without (dashed curves) third-order nonlinear polarizations are presented. Notice that introducing third-order polarization leads to minor corrections (within 5\%) in simulation results. Gale et $a l .{ }^{19}$ reported that without the cascade effect the thirdorder nonlinearity has only a minor influence on the OPA output. They discovered that, at the specific wavelength at which the cascade effect does take place, the nonlinearity greatly influences the duration of the output pulse, and they obtained good consistency between the experimental observation and the simulation results. In contrast, no cascade effect occurred over the whole wavelength tuning range in our OPA system. We therefore expected that no significant difference would be observed between the simulation results with and without consideration of third-order optical nonlinearity. In what follows, we therefore focus our discussion on the interplay between second-order nonlinearity and material dispersion.

Figure 5(a) shows output energies at several wavelengths. From 2.9 to $4.0 \mu \mathrm{m}$ the output energy drops slightly from 70 to $\sim 60 \mu \mathrm{J}$, corresponding to a photon conversion efficiency that varied from $10.4 \%$ to $11.3 \%$. The simulation results match the experimental data well. Almost perfect agreement in the output energy between the experimental and simulation results was achieved at both the second and the third amplification stages. For example, at $\lambda_{\text {idler }}=3.0 \mu \mathrm{m}$ the sum of the signal and the idler pulse energy measured at the site between mirrors DM3 and DM4 (Fig. 1) with only the pump beam of the first amplification stage was $\sim 2 \mu \mathrm{J}$, whereas that with the additional pump beam of the second amplification stage was $\sim 100 \mu \mathrm{J}$, reflecting a gain factor of 50 for the second amplification stage. In comparison, the corresponding simulation result shows a gain factor of 54 for the second amplification stage. Without the pump beam of the third amplification stage, the idler output pulse energy measured after DM6 was $\sim 30 \mu \mathrm{J}$, corresponding to a gain factor of 2.3 for the third amplification stage. The simulation yielded a gain factor of 2.7 for the third amplification stage. The limit in the output energy was caused mainly by the loss of dichroic mirrors DM2-DM6. Measured transmission coefficients of these components

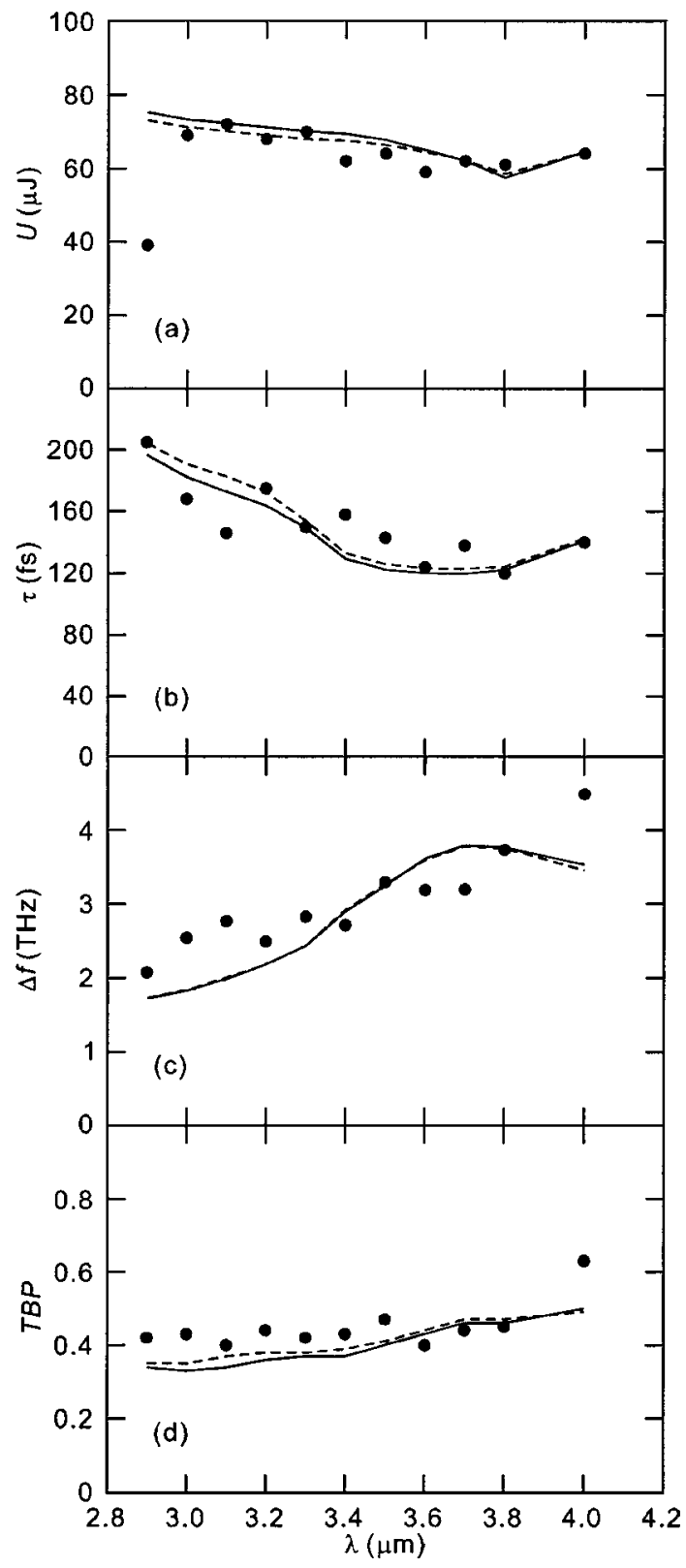

Fig. 5. Experimental and simulation results of the OPA system: (a) output energy $(U)$, (b) pulse width $(\tau)$, (c) spectral width $(\Delta f)$, (d) TBP. Experimental data are represented by filled circles. The simulation results are connected by curves: solid and dashed curves, results with and without third-order nonlinear polarization, respectively.

in the IR were $\sim 85 \%$. Future improvement in the coatings of these dichroic mirrors should improve the performance of the OPA system. The energy drop at $2.9 \mu \mathrm{m}$ was due to the absorption by water vapor, ${ }^{37}$ which was not taken into account in our simulation.

The output energy stability was $\sim 5 \%$. We attribute this excellent stability to three factors: First, the fluctuation in output energy of the Ti:sapphire regenerative amplifier system was $<\sim 2 \%$; this ensured the stability of the higher-order nonlinear optical mixing processes involved in white-light generation and optical parametric amplification. Second, the fine control of both the energy and the beam size of the $800-\mathrm{nm}$ pump beam has proved 
to be critical in producing a stable white-light seed beam that minimizes the instability of the OPA output. Third, the amplification gain of the third amplification stage showed some saturation, which further improves energy stability, ${ }^{5}$ as is illustrated in the simulation results described below.

Figure 5(b) shows the pulse duration (FWHM) of the idler wave extracted from the cross-correlation measurements. As the wavelength increased from 2.9 to $3.6 \mu \mathrm{m}$, the pulse duration decreased monotonically from $\sim 200$ to $\sim 130 \mathrm{fs}$. The pulse duration then stayed constant from 3.6 to $4.0 \mu \mathrm{m}$. The simulation results follow the experimental data well. The dependence on wavelength of the spectral width (FWHM) is shown in Fig. 5(c). Tuning the idler wavelength below $2.9 \mu \mathrm{m}$ resulted in strong distortion in the idler spectrum because of the absorption by water vapor mentioned above. An idler wavelength longer than $4.0 \mu \mathrm{m}$, however, encountered the absorption band of $\mathrm{CO}_{2}$ gas, ${ }^{37}$ which also resulted in distortion in the spectrum. From 3.0 to $3.8 \mu \mathrm{m}$ the spectral width increased with the wavelength from 2.5 to $3.7 \mathrm{THz}$ ( 83 to $123 \mathrm{~cm}^{-1}$ ), agreeing roughly with the simulation results. According to the results in Figs. 5(b) and 5(c), the values of the time-bandwidth product (TBP) were calculated; they are shown in Fig. 5(d). The TBP values increased slightly with wavelength and are close to the transform limit. This result is consistent with the illustration in Figs. 5(b) and 5(c) that the pulse duration of the idler wave decreases with output wavelength, whereas its spectral width shows an opposite dependence. These characteristics were also observed experimentally in other studies. ${ }^{10,12}$ We emphasize that the match in the output characteristics over the whole tunable wavelength range relies only on the simulation parameters at $3 \mu \mathrm{m}$ that were estimated from our experimental condition. No adjustment of these parameters is needed when one is tuning the output wavelength. Although our experimental characterization was performed at the third amplification stage, the good match between the experimental and simulation results strongly suggests that our simulation reflects actual details, especially in the photon conversion process, of our ultrafast OPA system.

\section{Coupling between Material Dispersion and Second- Order Optical Nonlinearity}

Understanding the interaction between material dispersion and optical nonlinearity during OPA has practical importance for the design and operation of OPA systems for ultrafast spectroscopy research. To investigate the interaction dynamics within the nonlinear optical crystal it is preferable to characterize the output of the OPA system while changing the crystal length. Two possible factors, however, prevent this characterization from being achieved experimentally. First, inasmuch as operating an ultrafast OPA system involves many complicated optical alignment and adjustment procedures, it is quite unreasonable to assume that all the experimental conditions are identical when nonlinear optical crystals with different thicknesses are used. Second, it is quite difficult and expensive to obtain nonlinear optical crystals that have identical optical characteristics (such as crystal orientation, coating properties, surface smoothness and flatness,

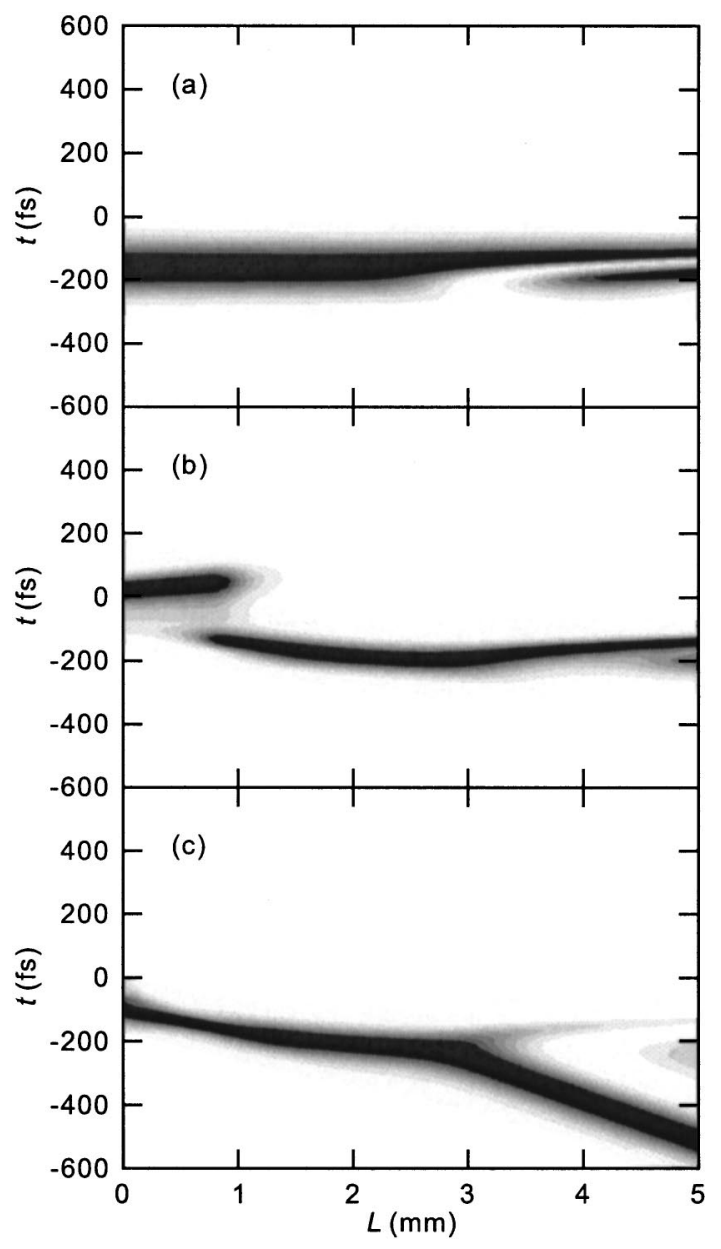

Fig. 6. Normalized pulse shape evolution of (a) pump, (b) signal, and (c) idler pulses within the KTA crystal; $L$, propagation distance.

and crystal uniformity) except the well-characterized thickness. Slight variations in these optical characteristics can result in large differences in output, because of the highly nonlinear dynamical behavior involved in ultrafast OPA. We therefore investigated the nonlinear dynamics in OPA based on our numerical simulation.

To investigate the interaction dynamics of an ultrafast OPA process numerically, we calculated the output characteristics for all the relevant pulses at different propagation distances inside the KTA crystal. Here we present the results at $\lambda_{\text {idler }}=3.0 \mu \mathrm{m}$ as a prototype example. To reveal the physical characteristics of ultrafast OPA we used photon density, instead of energy density, to describe the photon conversion process and its efficiency in this three-wave interaction. The simulation conditions were kept the same as those used to emulate experimental conditions. Figures 6 and 7 show the pulse shape and the spectral evolution, respectively, of the pump, the signal, and the idler waves. Figure 8 shows the photon densities of the three waves as they propagated inside the KTA crystal. According to Figs. 6-8, we can divide the evolution of the waves into three regions: pulse shaping $(<1$ $\mathrm{mm}$ ), stable amplification (roughly $1-3 \mathrm{~mm}$ ), and gain saturation $(>3 \mathrm{~mm})$. In the initial amplification region the pump pulse maintains its shape because no appreciable amount of its energy has been transferred to the 
signal and the idler waves. Owing to the initial chirp in the white-light seed pulse, the phase-matching condition chooses the proper section of the whole pulse shape that is to be amplified, resulting in a double-peaked pulse shape in the signal wave. This is consistent with the spectral evolution shown in Fig. 7, confirming that the phasematching mechanism automatically picks up the correct wavelength from the whole continuum spectrum for amplification. In addition, this effect gradually narrows the

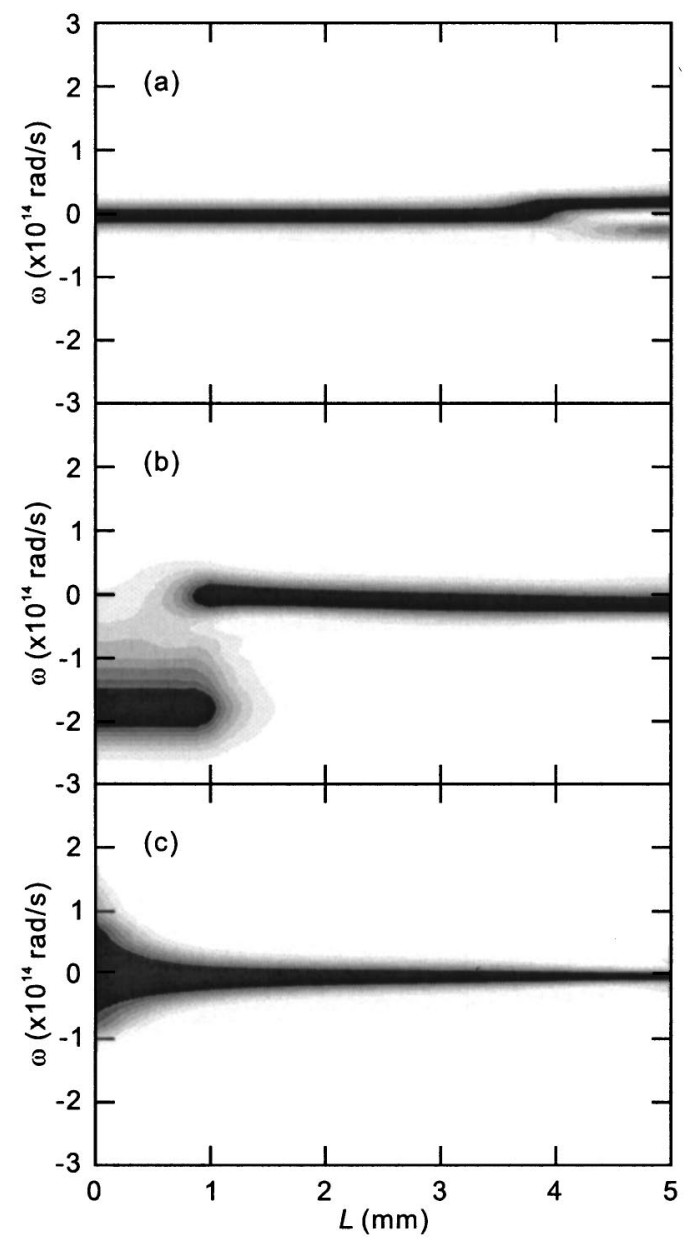

Fig. 7. Normalized spectrum evolution of (a) pump, (b) signal, and (c) idler pulses within the KTA crystal; $L$, propagation distance.

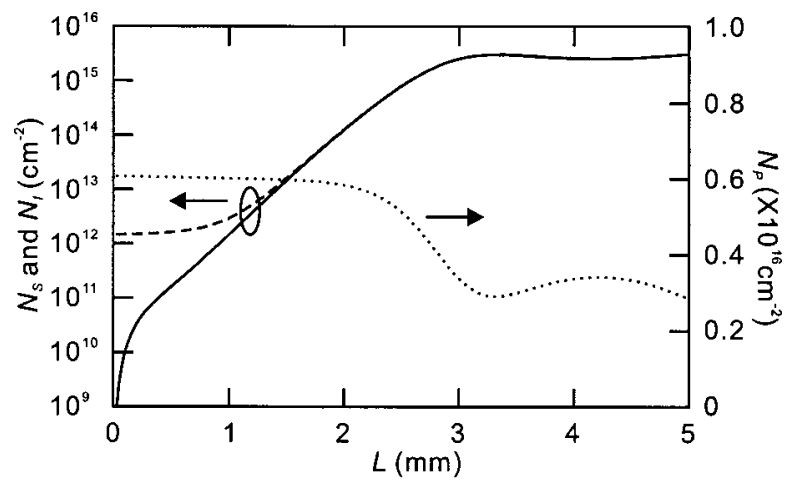

Fig. 8. Photon density of pump (dotted curve, $N_{P}$ ), signal (dashed curve, $N_{S}$ ), and idler (solid curve, $N_{I}$ ) waves inside the KTA crystal; $L$, propagation distance.

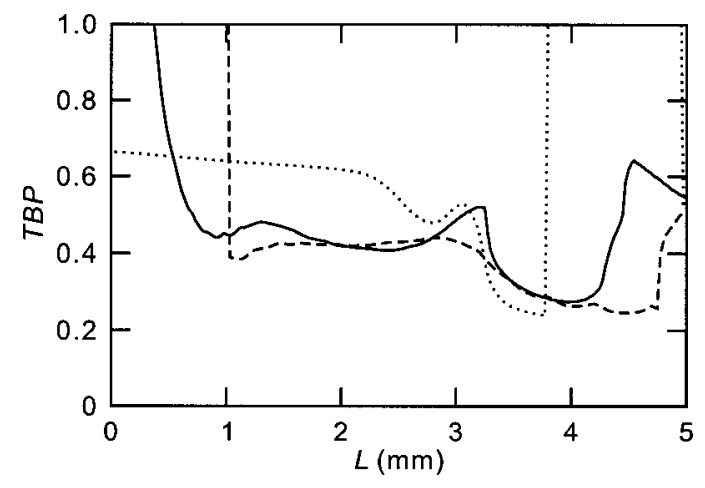

Fig. 9. TBP of pump (dotted curve), signal (dashed curve), and idler (solid curve) waves inside the KTA crystal; $L$, propagation distance.

spectral width of the idler wave as it propagates. The idler pulse grows at the temporal overlap between the pump and the signal pulses; it then follows the progression of the signal pulse, moving into a more negative temporal position. In this amplification region the signal wave grows slowly, while the idler wave has a much higher gain (Fig. 8). This amplification region is therefore called the pulse-shaping (PS) region, where the seed pulse plays an important role. After this region, both the signal and the idler waves reach stable pulse shape and spectra, though the idler wave shows a little narrowing because of the phase-matching condition. More importantly, both the signal and the idler waves are under linear gain amplification (Fig. 8). This may be the reason why their pulse shapes and spectra are sustained. This stable-amplification (SA) region extends from $\sim 1$ to $\sim 3$ $\mathrm{mm}$. Photon densities of the signal and the idler waves merge gradually in the $\mathrm{SA}$ region. The location where their photon densities meet marks the end of the influence of the seed pulse. Beyond $3 \mathrm{~mm}$, the energy of the signal and the idler waves becomes large enough to deplete the pump wave, leading to splitting of the pump pulse, gain saturation, and low conversion efficiency. The signal and the idler pulses are so intense that they start to regenerate the pump wave at a different temporal position. The signal and the idler pulses then become depleted and are split too. This behavior corresponds to the highly distorted part of the plot in Fig. 7(a). This gain saturation (GS) region can be easily identified in Fig. 8 as the area where the energy flows back and forth among these three waves. Notice that the idler pulse separates temporally from the pump and the signal pulses at the beginning of the gain-saturation region. The analysis of the OPA process can be further elucidated by evolution of the values of the TBP in Fig. 9. Notice that only in the $\mathrm{SA}$ region do the TBP values of both the signal and the idler pulses remain at $\sim 0.4$, that is, very close to the transform-limited value. The extremely large TBP values of the signal wave before $\sim 1 \mathrm{~mm}$ and of the idler wave after $\sim 3.8 \mathrm{~mm}$ are due to pulse splitting in the PS and the GS regions, respectively.

What is the origin of the SA region? To answer this question, one has to consider the interaction between material dispersion and the second-order optical nonlinearity. As pump, signal, and idler waves propagate inside the nonlinear optical crystal, they are temporally sepa- 
rated because of a GVM and are simultaneously broadened owing to GVD. The overall effect is smaller gain for both the signal and idler waves. Because of these two effects, one can estimate the effective interaction length of the three waves by calculating the propagation distance where the largest temporal separation among these three waves is equal to the pulse duration of the pump pulse. Here this simple estimation leads to an interaction length of $\sim 2 \mathrm{~mm}$, which is shorter than the total length of the PS and SA regions $(\sim 3 \mathrm{~mm})$, indicating that efficient amplification is not limited by GVM and GVD, in agreement with a previous observation. ${ }^{9}$ Physically, the nonlinear polarizations involved in the OPA process act as source terms in the nonlinear coupling equations, Eqs. (2)-(4), and temporally pull the three waves together. It is this pulling mechanism that preserves the exponential gain in the SA region. For example, in the beginning, the intense pump wave produces two strong nonlinear polarizations, $P_{S}{ }^{\mathrm{NL}}$ and $P_{I}^{\mathrm{NL}}$, at the temporal overlap between the pump wave and the signal and idler waves. These two source terms then amplify the signal wave and generate an idler wave at the temporal positions of $P_{S}{ }^{\mathrm{NL}}$ and $P_{I}^{\mathrm{NL}}$, respectively. The nonlinear polarization for the pump wave, $P_{P}{ }^{\mathrm{NL}}$, however, is small because of the weak signal and idler waves and has little influence on the pump wave. The overall effect of the nonlinear interaction is to pull the signal and the idler pulses toward the pump pulse. This process overcomes the effects of the GVM and GVD and thus prolongs the interaction length. This situation breaks down when the pump pulse is depleted near the GS region. The energy of the signal and the idler waves becomes so large that the effect of the source terms, $P_{S}{ }^{\mathrm{NL}}$ and $P_{I}^{\mathrm{NL}}$, decreases relatively. At the same time, $P_{P}{ }^{\text {NL }}$ now becomes large enough to influence the temporal position of the pump pulse. The pulling mechanism therefore weakens, and the effects of the GVM and GVD gradually emerge, separating these three waves temporally and halting the amplification process, as shown for the results beyond $3 \mathrm{~mm}$ in Figs. 6-8. The pulling mechanism has also been recognized in other research. ${ }^{6,9,38}$ One of the problems facing designers of ultrafast OPA systems lies in choosing an appropriate crystal thickness. The nonlinear optical crystal should be thick enough to produce large output energy and thin enough to prevent pulse distortion and splitting. Because of the almost perfect match between the experimental and simulation results in our case, our simulation can be used to optimize the crystal thickness. ${ }^{23}$

\section{Effects of Fluctuation of the Pump Intensity}

To study its influence on OPA, we simulated variations in the pump intensity. One can adjust the intensity either by varying the energy density while keeping the pulse width constant or by varying the pulse width while maintaining the energy density. For this study we chose the former procedure. The reasons for this choice are threefold. First, a study of this fluctuation can reveal the dependence of the instability in the output characteristics on fluctuations in the pump energy. Second, the dependence of the output characteristics on the pump energy can show OPA designers how to distribute pump energy properly in different amplification stages and show spec- troscopic experimentalists whether a simple increase in the pump pulse's energy can generate more-intense OPA output while maintaining the pulse quality. Third, in actual experiments it is normally much easier to vary the pump energy density than the pulse width. This is so because varying the pulse width is inevitably accompanied by variation in the chirp condition, which changes the characteristics of the output pulse.

The pump intensity in the actual OPA system (52 $\mathrm{GW} / \mathrm{cm}^{2}$ ) was used as the reference, $I_{P 0}$. Figure 10 shows the variations in pulse duration and in photon density $\left(\Delta \tau / \tau_{0}\right.$ and $\left.\Delta N / N_{0}\right)$ of the signal and the idler waves at $3 \mathrm{~mm}$ caused by variation of the pump intensity $\left(\Delta I_{P} / I_{P 0}\right)$ from $-30 \%$ to $+30 \%$. $\tau_{0}$ is the duration of the output pulse and $N_{0}$ is the photon density with the reference pump's intensity. The tangential line in Fig. 10(a) has a slope equal to 1 . For $\left|\Delta I_{P} / I_{P 0}\right|<5 \%, \Delta \tau / \tau_{0}$ of the idler wave is almost identical to $\Delta I_{P} / I_{P 0}$, whereas the pulse duration of the signal wave experiences much smaller variation, indicating that the pulse shape of the signal wave is more tolerant of fluctuations in pump energy. This suggests that one has to put more effort into maintaining the stability of the pump pulse's energy when one uses the idler pulse in spectroscopic measurements. According to Fig. 6(c), the duration of idler pulse has a more sensitive dependence on the propagation distance in the crystal than does the duration of the signal pulse. This may explain why the duration of the idler pulse is more sensitive to fluctuations in pump intensity. The highly distorted behavior of $\Delta \tau / \tau_{0}$ for large $\Delta I_{P} / I_{P}$ is due to pulse splitting under higher pump intensity. The pulse splitting is caused by shortening of the SA region with higher pump intensity, which causes the amplifica-

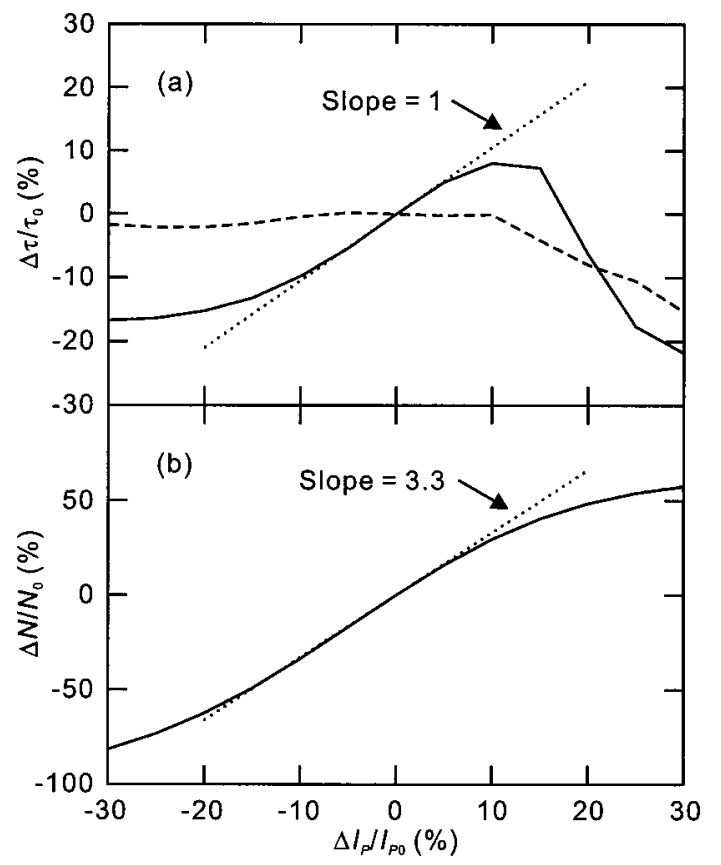

Fig. 10. Variations of (a) pulse duration $\left(\Delta \tau / \tau_{0}\right)$ and (b) photon density $\left(\Delta N / N_{0}\right)$ of signal (dashed curves) and idler (solid curves) waves at $3 \mathrm{~mm}$ caused by variation of the pump intensity $\left(\Delta I_{P} / I_{P 0}\right): \tau_{0}$, pulse duration; $N_{0}$, photon density with reference pump intensity $\left(I_{P 0}\right)$. Dotted curves, tangential line of the data at $I_{P 0}\left(\Delta I_{P} / I_{P 0}=0\right)$. 
tion to enter the GS region before $3 \mathrm{~mm}$. Notice that the fluctuation in the pump intensity has identical influence on the photon densities of the signal and the idler waves, as shown in Fig. 10(b). This may be so because the photon densities of the signal and idler waves merge before $L=3 \mathrm{~mm}$ (Fig. 8). $\left|\Delta N / N_{0}\right|$ for higher pump intensity is slightly less than that for lower pump intensity, which is also a result of shortening of the SA region. The tangential line in Fig. 10(b) has a slope equal to 3.4. For $\left|\Delta I_{P} / I_{P 0}\right|<5 \%, \Delta N / N_{0}$ of both the signal and the idler waves is almost three times $\Delta I_{P} / I_{P 0}$.

We can draw two conclusions from the analysis above. First, the variations of the pulse duration and the output energy are equal to or larger than that of the pump intensity, imposing a stricter requirement for stability of the pump laser in the design of ultrafast OPA systems, as the variation in pulse duration may make interpretation in the time resolution of ultrafast spectroscopic measurements difficult. Second, a linear dependence of the output energy on the pump intensity is not expected from the nonlinear optical processes in an OPA system, which can be explained by the fact that some saturation in the OPA process occurs at $3 \mathrm{~mm}$. This analysis demonstrates the importance of using an appropriate simulation tool to help in identifying the optimal design parameters of OPA systems.

\section{E. Unrealistic White-Light Seed Pulse}

An artificially generated white-light seed was usually used in numerical investigations of ultrafast OPA systems. For example, flat-topped spectra were used by Gale et al. ${ }^{20}$ and Reisner and Gutmann ${ }^{21}$ to represent the white-light continuum. The spectral intensity was assumed to be constant within a certain bandwidth and to be zero outside this range. Pang et al. ${ }^{23}$ used a chirped super-Gaussian pulse for the white-light continuum. ${ }^{22}$ As shown in Fig. 4, the actual white-light seed pulse is quite different from the artificial pulses mentioned above. Therefore it is important to understand how an unrealistic seed pulse influences the simulation results. Based on the almost perfect match with the experimental results that occurs when the actual electric field of the seed pulse is used in the simulation, we expect that our simulation can shed light on the difference in the output characteristics and the pulse evolution dynamics when different seed signal pulses are used.

We chose to carry out our simulation by using two different artificial white-light seed pulses. In the first case, pulses with flat-topped spectra were used as the seed pulses. The spectral width was again chosen to be the tuning range of our OPA system (from 1 to $1.1 \mu \mathrm{m}$ ), and the group delay was chosen to be the measured result described in Section 2. In the second case, pulses with the actual spectral distribution shown in Fig. 4 but with the phase disregarded (i.e., chirpless pulses) were used as the seed pulses. In both cases we scaled the intensity of the seed pulses to achieve the same total output energy as that of the first amplification stage at $\lambda_{\text {idler }}=3.0 \mu \mathrm{m}$, similar to the case for the actual white-light seed pulse described above. The simulation was then performed at other idler wavelengths without any further adjustment in the simulation parameters. The simulation results

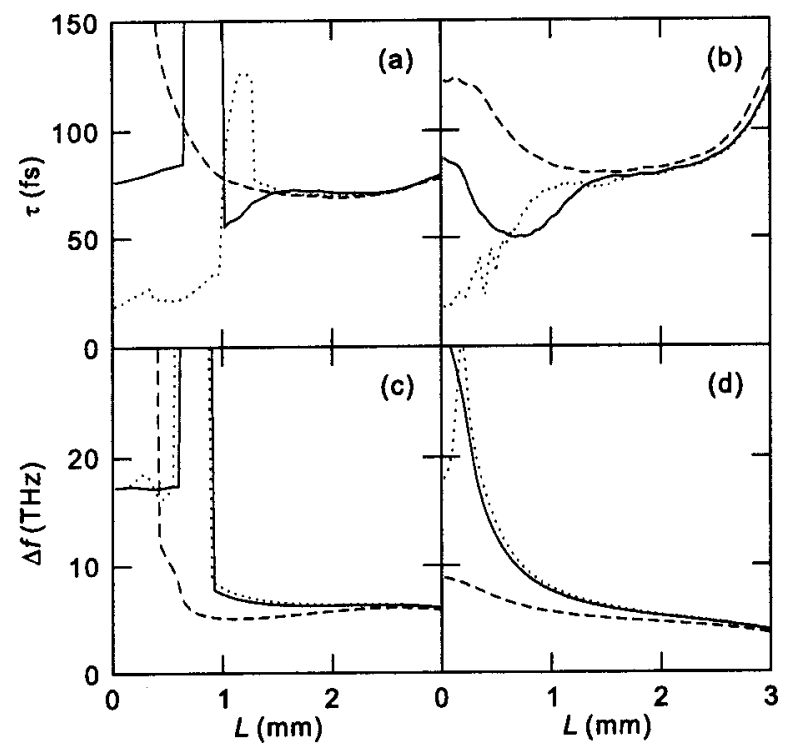

Fig. 11. Evolution of the pulse duration and the spectral width of signal and idler waves inside the OPA crystal at $\lambda_{\text {idler }}$ $=3 \mu \mathrm{m}$ : pulse durations of (a) the signal and (b) the idler waves; spectral widths of (c) the signal and (d) the idler waves. Simulation results with the real, flat-topped, and chirpless white-light seed pulses are represented by solid, dashed, and dotted curves, respectively.

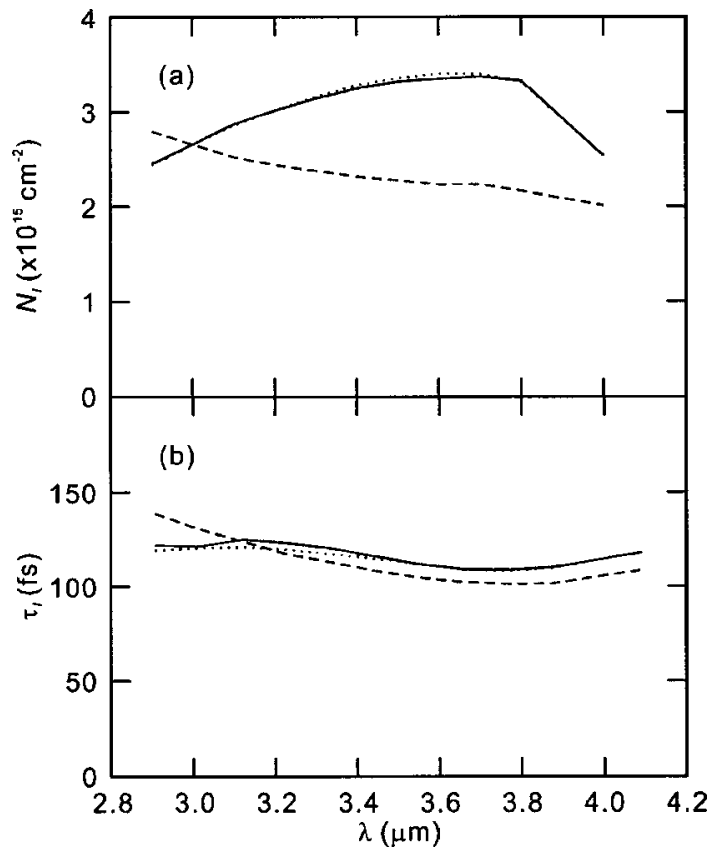

Fig. 12. (a) Output photon density and (b) pulse duration of the idler wave at several wavelengths with the real (solid curves), flat-topped (dashed curves), and chirpless (dotted curves) whitelight seed pulses.

were then compared with those obtained with the actual white-light seed pulse. Inasmuch as the total output energy was intentionally unchanged, the comparison was concentrated on the evolution of pulse quality at $\lambda_{\text {idler }}$ $=3.0 \mu \mathrm{m}$ (Fig. 11) and on the output characteristics at different wavelengths (Fig. 12). The sudden changes in pulse duration and spectral width shown in Fig. 11 indi- 
cate pulse splitting (similar to that shown in Fig. 6). Notice that the evolution dynamics in the PS region are totally different for the three white-light seed pulses, indicating that the true dynamics in the pulse-shaping process is lost if unrealistic white-light seed pulses are used. The pulse duration and the spectral width gradually merge as the amplification proceeds. This is a natural outcome because we have intentionally scaled the intensity of the seed pulses to match the simulation results at this wavelength. We discovered, however, that at other idler wavelengths the simulation results with the flat-topped white-light seed pulse show great deviations in both the output photon density and the duration of the output idler pulse (Fig. 12). The simulation with the chirpless white-light seed pulse shows almost identical results to the ones obtained with the actual white-light seed pulse, however. This may be due to the following two factors: First, the chirpless white-light seed pulse has an actual spectral distribution. Second, the difference in its phase from the actual phase is compensated for by adjustment of the temporal delay between the pump and seed pulses when the output idler wavelength is varied.

In sum, the flat-topped spectral shape is independent of the amplitude variation across the tunable wavelength range of the signal wave (Fig. 4). Even when its intensity was scaled to match the simulation results at a specific wavelength, the same simulation parameters did not produce correct results at other wavelengths. In other words, one has to adopt different scaling factors for different wavelengths. Apparently such adjustment in the energy density of the seed pulse is unphysical, and there is no way to determine these factors theoretically. The intensity of a chirpless white-light seed pulse also needs to be adjusted away from the experimental value to match the simulation results. Moreover, the temporal delays of the pump pulse to maximize the output energy at all idler wavelengths are the same, unlike for the actual OPA system. With correct chirp information on the white-light seed pulse, our simulation can thus provide a useful tool for designing a computer-controlled ultrafast OPA system.

\section{CONCLUSIONS}

We have succeeded in generating intense pulses (up to $\sim 70 \mu \mathrm{J}$ ) with wavelengths tuned from 2.9 to $4.0 \mu \mathrm{m}$ and time-bandwidth product values close to the transform limit. A numerical simulation has been developed based on this system. In this simulation, the phase-matching condition and the actual electric field of the white-light seed pulse were properly accounted for. No artificial scaling of the pump and seeded intensities was needed to match the experimental results. The almost perfect match of the simulation with the experimental results demonstrates that our simulation can account for many practical aspects of an optical parametric amplification system and that it can provide a powerful analytical tool with which to investigate the sophisticated interaction between material dispersion and optical nonlinearity. Three amplification phases, i.e., pulse shaping, stable amplification, and gain saturation, have been recognized and have made the analysis easier. The seed pulse was found to stimulate energy transfer among pump, signal, and idler waves and gradually lost its original characteristics as it gained energy from the intense pump beam. According to our simulation results, fluctuation in the pump intensity will be amplified during OPA, which imposes a stricter requirement for stability of the pump laser. It has been recognized that simulations with unrealistic white-light seed pulses are incapable of revealing the actual interaction dynamics in OPA and moreover that a nonphysical adjustment in the pump and the seed intensities is needed. Our research has provided both qualitative and quantitative descriptions of the nonlinear optical interaction in ultrafast OPA systems. Besides being interesting for nonlinear optics, it should also provide helpful information for improving the performance of existing and prospective OPA systems.

\section{ACKNOWLEDGMENTS}

This research is supported by the National Science Council of Taiwan (grant 92-2112-M-002-011) and by the Academia Sinica of Taiwan.

J.-K. Wang's e-mail address is jkwang @po.iams.sinica.edu.tw.

\section{REFERENCES}

1. G. A. Reider and T. F. Heinz, "Second-order nonlinear optical effects at surfaces and interfaces: recent advances," in Photonic Probes of Surfaces, P. Halevi, ed. (North-Holland, Amsterdam, 1995), pp. 413-478.

2. Y. R. Shen, "Sum frequency generation for vibrational spectroscopy: applications to water interfaces and films of water and ice," Solid State Commun. 108, 399-406 (1998), and references therein.

3. Y. R. Shen, The Principles of Nonlinear Optics (Wiley, New York, 1984).

4. M. H. Dunn and M. Ebrahimzadeh, "Parametric generation of tunable light from continuous-wave to femtosecond pulses," Science 286, 1513-1517 (1999).

5. V. Petrov, F. Rotermund, and F. Noack, "Generation of highpower femtosecond light pulses at $1 \mathrm{kHz}$ in the midinfrared spectral range between 3 and $12 \mu \mathrm{m}$ by secondorder nonlinear processes in optical crystals," J. Opt. A: Pure Appl. Opt. 3, R1-R19 (2001).

6. R. Danielius, A. Piskarskas, A. Stabinis, G. P. Banfi, P. Di Trapani, and R. Righini, "Traveling-wave parametric generation of widely tunable, highly coherent femtosecond light pulses," J. Opt. Soc. Am. B 10, 2222-2232 (1993).

7. M. K. Reed, M. K. Steiner-Shepard, M. S. Armas, and D. K. Negus, "Microjoule-energy ultrafast optical parametric amplifiers," J. Opt. Soc. Am. B 12, 2229-2236 (1995).

8. K. R. Wilson and V. V. Yakovlev, "Ultrafast rainbow: tunable ultrashort pulses from a solid-state kilohertz system," J. Opt. Soc. Am. B 14, 444-448 (1997).

9. V. Petrov, F. Noack, and R. Stolzenberger, "Seeded femtosecond optical parametric amplification in the mid-infrared spectral region above $3 \mu \mathrm{m}$," Appl. Opt. 36, 1164-1172 (1997).

10. G. M. Gale, G. Gallot, F. Hache, and R. Sander, "Generation of intense highly coherent femtosecond pulses in the midinfrared," Opt. Lett. 22, 1253-1255 (1997).

11. U. Emmerichs, S. Wouterson, and H. J. Bakker, "Generation of intense femtosecond optical pulses near $3 \mu \mathrm{m}$ with a kilohertz repetition rate," J. Opt. Soc. Am. B 14, 1480-1483 (1997).

12. S. Cussat-Blanc, A. Ivanov, D. Lupinski, and E. Freysz, " $\mathrm{KTiOPO}_{4}, \mathrm{KTiOAsO}_{4}$, and $\mathrm{KNbO}_{3}$ crystals for midinfrared femtosecond optical parametric amplifiers: analy- 
sis and comparison,” Appl. Phys. B (Suppl.) 70, S247-S252 (2000), and references therein.

13. M. Nisoli, S. Stagira, S. De Slivestri, O. Svelto, G. Valiulis, and A. Varanavicius, "Parametric generation of high-energy 14.5-fs light pulses at $1.5 \mu \mathrm{m}$," Opt. Lett. 23, 630-632 (1998).

14. S. R. Greenfield and M. R. Wasielewski, "Near-transformlimited visible and near-IR femtosecond pulses from optical parametric amplification using type II $\beta$-barium borate," Opt. Lett. 20, 1394-1396 (1995).

15. D. E. Gragson, D. S. Alavi, and G. L. Richmond, "Tunable infrared laser system based on parametric amplification in KTP with a Ti:sapphire amplifier,” Opt. Lett. 20, 1991-1993 (1995).

16. J. A. Armstrong, N. Bloembergen, J. Ducuing, and P. S. Pershan, "Interactions between light waves in a nonlinear dielectric," Phys. Rev. 127, 1918-1939 (1962).

17. S. A. Akhmanov, A. S. Chirkin, K. N. Drabovich, A. I. Kovrigin, R. V. Khokhlov, and A. P. Sukhorukov, "Nonstationary nonlinear optical effects and ultrashort light pulse formation," IEEE J. Quantum Electron. QE-4, 598-605 (1968).

18. H. J. Bakker, P. C. M. Planken, and H. G. Muller, "Numerical calculation of optical frequency-conversion processes: a new approach,” J. Opt. Soc. Am. B 6, 1665-1672 (1989), and references therein.

19. G. M. Gale, M. Cavallari, and F. Hache, "Femtosecond visible optical parametric oscillator," J. Opt. Soc. Am. B 15, 702-714 (1998).

20. G. M. Gale, F. Hache, and M. Cavallari, "Broad-bandwidth parametric amplification in the visible: femtosecond experiments and simulations," IEEE J. Sel. Top. Quantum Electron. 4, 224-229 (1998).

21. S. Reisner and M. Gutmann, "Numerical treatment of UVpumped, white-light-seeded single-pass noncollinear parametric amplifiers," J. Opt. Soc. Am. B 16, 1801-1813 (1999).

22. D. Pang, R. Zhang, and Q. Wang, "Theoretical analysis of noncollinear phase-matched optical parametric amplifier seeded by a white-light continuum," Opt. Commun. 196, 293-298 (2001).

23. J.-C. Wang and J.-K. Wang, "Femtosecond optical parametric amplifiers with collinear phase matching: experiments and full simulation," presented at the Photonics West, Lasers and Applications in Sciences and Engineering Symposium, San Jose, Calif., January 25-31, 2003.

24. W. Plass, H. Rottke, W. Heuer, G. Eichhorn, and H. Zacharias, "Surface sum-frequency mixing for auto- and crosscorrelation of ultrashort UV and IR pulses," Appl. Phys. B 54, 199-201 (1992).

25. E. J. Canto-Said, P. Simon, C. Jordan, and G. Marowsky, "Surface second-harmonic generation in $\mathrm{Si}(111)$ for autocor- relation measurements of 248-nm femtosecond pulses," Opt. Lett. 18, 2038-2040 (1993).

26. H. P. Li, C. H. Kam, Y. L. Lam, F. Zhou, and W. Ji, "Nonlinear refraction of undoped and $\mathrm{Fe}$-doped $\mathrm{KTiOAsO}_{4}$ crystals in the femtosecond regime," Appl. Phys. B 70, 385-388 (2000)

27. D. L. Fenimore, K. L. Schepler, U. B. Ramabadran, and S. R. McPherson, "Infrared corrected Sellmeier coefficients for potassium titanyl arsenate," J. Opt. Soc. Am. B 12, 794-796 (1995).

28. W. H. Press, S. A. Teukolsky, W. T. Vetterling, and B. P. Flannery, Numerical Recipes in C, 2nd ed. (Cambridge U. Press, New York, 1992).

29. P. Hamm, R. A. Kaindl, and J. Stenger, "Noise suppression in femtosecond mid-infrared light sources," Opt. Lett. 25, 1798-1800 (2000)

30. R. R. Alfano, The Supercontinuum Laser Source (SpringerVerlag, New York, 1989).

31. R. L. Fork, C. V. Shank, C. Hirlimann, R. Yen, and W. J. Tomlinson, "Femtosecond white-light continuum pulses," Opt. Lett. 8, 1-3 (1983).

32. G. Yang and Y. R. Shen, "Spectral broadening of ultrashort pulses in a nonlinear medium," Opt. Lett. 9, 510-512 (1984).

33. S. A. Diddams, H. K. Eaton, A. A. Zozulya, and T. S. Clement, "Amplitude and phase measurements of femtosecond pulse splitting in nonlinear dispersive media," Opt. Lett. 23, 379-381 (1998)

34. J. M. Dudley and S. Coen, "Numerical simulations and coherence properties of supercontinuum generation in photonic crystal and tapered optical fibers," IEEE J. Quantum Electron. 8, 651-659 (2002)

35. X. Gu, L. Xu, M. Kimmel, E. Zeek, P. O'Shea, A. P. Shreenath, R. Trebino, and R. S. Windeler, "Frequencyresolved optical gating and single-shot spectral measurements reveal fine structure in microstructure-fiber continuum," Opt. Lett. 27, 1174-1176 (2002)

36. J. M. Dudley, X. Gu, L. Xu, M. Kimmel, E. Zeek, P. O’Shea, R. Trebino, S. Coen, and R. S. Windeler, "Cross-correlation frequency resolved optical gating analysis of broadband continuum generation in photonic crystal fiber: Simulations and experiments," Opt. Express 10, 1215-1221 (2002), http://www.opticsexpress.org.

37. W. L. Wolfe and G. J. Zissis, The Infrared Handbook (Environmental Research Institute of Michigan, Detroit, Mich. 1993).

38. M. Nisoli, S. De Slivestri, V. Magni, O. Svelto, R. Danielius, A. Piskarskas, G. Valiulis, and A. Varanavicius, "Highly efficient parametric conversion of femtosecond Ti:sapphire laser pulses at $1 \mathrm{kHz}$," Opt. Lett. 19, 1973-1975 (1994). 